\title{
Study on the Improved Parallel Braking Control Strategy of Range-extended Electric Commercial Vehicle
}

\author{
Xu Shi-wei ${ }^{1, a}$, Wang Dong-liang ${ }^{1, b}$ and $\mathrm{He}$ Yi-lin $^{1, c}$ \\ ${ }^{1}$ School of automobile, Chang'an University, Shaanxi Xi'an 710064, China. \\ axushiweide2008@163.com, ${ }^{\mathrm{b}}$ 1063670691@qq.com, ${ }^{\mathrm{c}}$ 553571576@qq.com
}

Keywords: automotive engineering, range-extended electric commercial vehicle, improved parallel braking control strategy, braking energy recovery

Abstract. For the problems of electric vehicle regenerative braking, an improved parallel braking control strategy model at Cruise and Matlab/Simulink co-simulation environment was established under the braking constraints. Then UDC cycle is used to verify the performance of brake control strategy. The simulation results show that the improved parallel braking control strategy can optimize the brake force distribution, and maximize the braking energy recovery under ensuring the best braking performance, so the braking performance is improved and meanwhile the vehicle economy is largely enhanced.

\section{Introduction}

As the conventional vehicles consuming a large amount of fossil energy, conventional vehicles have severely caused environmental crises and energy crises ${ }^{[1]}$. The electric vehicle technology, as a new technology of obvious advantages involving energy conservation, emission reduction and environment protection, has been attracting the attention of governments, enterprises and research institutions. Regenerative braking is a kind of electric vehicle technologies to recycle the braking energy by motor when vehicle braking. So the regenerative braking has a positive effect on increasing the electric vehicle mileage and improving energy utilization rate. However, at the present stage, the research on the regenerative braking was mostly concentrated on the electric passenger car rather than the commercial vehicle $^{[2]}$. In this paper, the extended-range electric commercial vehicle was studied which can recycle the braking energy like the electric passenger car. The extended-range electric vehicle has certain key features such as high energy utilization rate, energy conservation and emission reduction ${ }^{[3]}$. The performance of improved parallel braking control strategy is studied by co-simulation methods in this paper.

\section{Restraints of improved parallel braking on extended-range electric commercial vehicle}

\section{Braking force distribution requirements of ECE regulation}

The distribution of the front and rear braking force will affect the direction stability of the vehicle and the adhesive condition utilization when braking. Locking the front and rear wheels simultaneously is a kind of ideal conditions. In addition, the braking force distribution has to meet the corresponding braking laws and regulations to ensure the braking efficiency. For dual-axle vehicles, there are clear regulations on the distribution of the front and rear brake force by ECE R13 regulation and GB 12676-1999. The braking intensity must meet the requirement $\mathrm{z} \geq 0.1+0.85(\varphi-0.2)$ when the adhesive coefficient $\varphi$ is between 0.2 and $0.8^{[4]}$.

\section{The restrictions of motor}

In a constant rotating speed, the regenerative braking torque is related to the motor generating power and efficiency ${ }^{[5]}$. Due to the limit of power generation, the maximum regenerative braking torque cannot exceed the generating capacity of the motor. When the vehicle brakes in a low speed, the motor regenerative braking force will become small with the low motor speed. In order to ensure the braking safety, the regenerative braking speed threshold is set as $500 \mathrm{r} / \mathrm{min}$. The regenerative braking will terminate when the motor speed is lower than the threshold ${ }^{[6]}$. So the regenerative braking torque 
can be expressed as:

$$
T_{\text {reg }}=\lambda(n) T_{m}
$$

Where $T_{m}$ is the motor torque in $\mathrm{N} \bullet \mathrm{m} ; \lambda(n)$ is the correction factor associated with the motor speed:

$$
\lambda(n)= \begin{cases}0 & n \leq 500 r / \min \\ 1 & n>500 r / \min \end{cases}
$$

\section{The restrictions of battery}

The charging current and power are limited by the maximum allowable charging current and power of the battery respectively ${ }^{[7]}$. Besides, both over charge and discharge have adverse effect on battery. Thus, the battery SOC range has to be restricted. The SOC operating range for the lithium-ion battery is $30 \% \sim 90 \%$ in this paper. When SOC is higher than $90 \%$, the regenerative braking stopped. Therefore, the charging power $P_{b a t}$ can be expressed as:

$$
P_{b a t}=\left(U_{o c}+I R\right) I
$$

Where $U_{O C}$ is the open circuit voltage in $\mathrm{V}$, and $R$ is the internal resistance of the battery in $\Omega$. $I$ is the current of the charging current in A, which is calculated as:

$$
I=I_{0} \mathrm{e}^{-\sigma \mathrm{t}}
$$

Where $I_{0}$ is the maximum initial charging current in A, while $\sigma$ is the attenuation coefficient, which is also called as the charge acceptance ratio.

\section{Model of improved parallel braking control strategy}

\section{Principle of improved parallel braking control strategy}

As shown in Fig. 1, the distributions of regenerative braking force and mechanical friction braking force are according to the fixed proportion. Therefore, there is less change on the mechanical braking system. The improved parallel braking system with additional control function is suitable for electric vehicle because it has simple structure and low cost.

The control process of improved parallel braking system is shown in Fig.2. The motor controller calculates the electric braking torque which is determined by the hydraulic master cylinder pressure. The motor braking torque is also based on the driver's braking command. The electric braking force is the function of the motor speed, which is decided by the distribution proportion such as the braking force, the motor's torque characteristics, the driver's perception and the road adhesion limit.

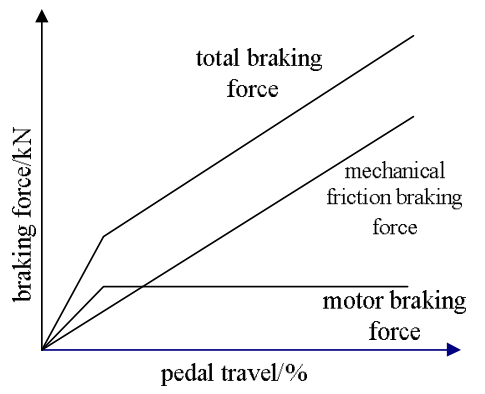

Fig.1 Principle of improved parallel braking force distribution strategy 


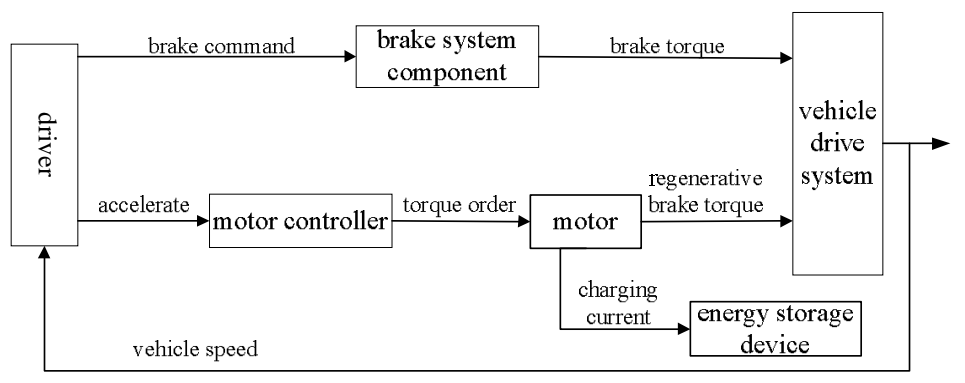

Fig.2 Principle of improved parallel braking control strategy

\section{The distribution strategy of improved parallel braking force}

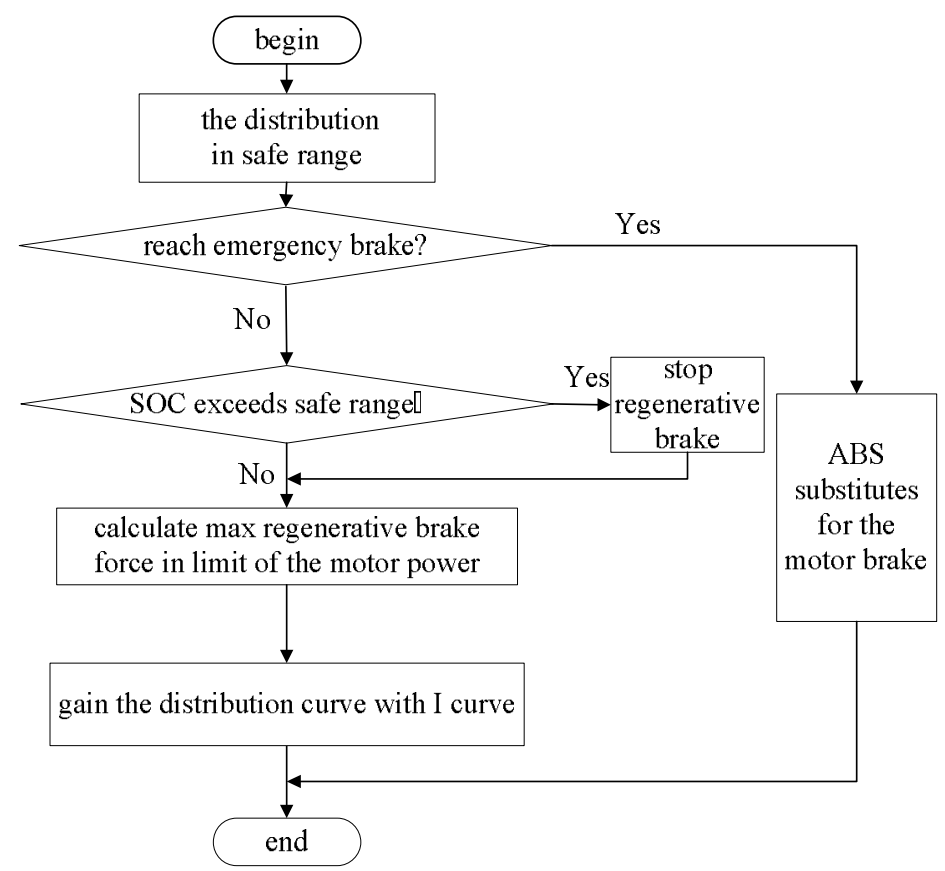

Fig.3 Flow chart of the distribution strategy

For the improved parallel braking control strategy, the braking force distribution is mainly based on the braking intensity. The system can recycle the braking energy as much as possible on the premise of vehicle stability. Specific allocation method is as follows:

In mild braking $(\mathrm{z} \leq 0.1)$ and moderate braking $(0.1<\mathrm{z} \leq 0.7)$, both regenerative braking force and pneumatic braking force were activated to recycle as much braking energy as possible on the foundation of braking performance. In the progress, the regenerative braking force is guaranteed firstly. In mild braking and the early stage of the coasting braking, the motor braking force is adjusted to increase with the pneumatic braking adjusting to decrease. Because the vehicle speed is usually higher in mild braking, mild braking is the main stage of energy recovery with the high efficiency of the motor. In moderate braking intensity, the motor works in best feedback efficiency areas and the friction braking fills up the rest braking force. In severe braking $(\mathrm{z}>0.7)$ and emergency braking, the regenerative braking force should be reduced or terminated to prevent the wheel from locking and ensure the braking stability.

Since the heavy commercial vehicle is rear wheel drive in this paper, the distribution strategy is mostly involved in the rear wheel. The specific process is shown in figure 3:

Step 1: According to the ECE regulation and braking stability requirements, the initial distribution ratio of the vehicle's front and rear wheel braking force is gotten in the boundary of the safe braking force distribution.

Step 2: According to braking intensity, the braking state whether or not current braking is a emergency brake is made. If yes, turns off the motor brake and the ABS act on; otherwise, turns to the next step.

Step 3: The regenerative braking should be limited by battery SOC and battery active charging area. When the SOC exceeds, the regenerative braking is terminated to ensure the charging safety. 
Step 4: The maximum regenerative braking force provided by the motor is calculated after considering the restrictions of the motor and the battery.

Step 5: Ultimately, the braking force distribution of the improved parallel braking strategy is obtained. In the process, when braking intensity $\mathrm{z}>0.7$, ABS substitutes for the motor braking, then the braking distribution curve meets the ideal curve-I curve.

\section{Improved parallel braking model based on Cruise and Matlab/Simulink}

The simulation model of the improved parallel braking strategy is showed in Fig.4. First, the strategy should be built in Matlab/Simulink, then it is convert into DLL file. Next the DLL file is loaded into the Cruise vehicle model by Cruise Interfaces, and the input and output interface bus should be connected at last.

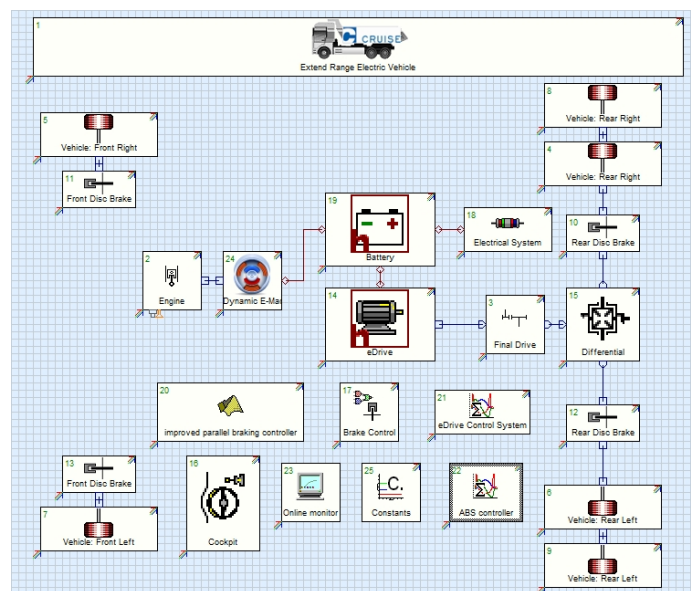

Fig.4 Improved parallel braking model based on Cruise and Matlab/Simulink

\section{Results and analysis}

In order to verify the performance of braking control strategy, the UDC cycle is adopted in the simulation model. Then the simulation results are compared with the non-energy recovery strategy.

Fig. 5 shows that under different velocity, the actual velocity can rapidly and accurately follow the desired velocity except small amplitude at the end of the acceleration. Fig.6 shows that when driving, the motor torque is positive to overcome the driving resistance; on the contrary, when braking, the motor torque is negative and produce negative mechanical power to recycle the braking energy. The motor torque and the mechanical efficiency proves the availability of control strategy in the driving cycle.

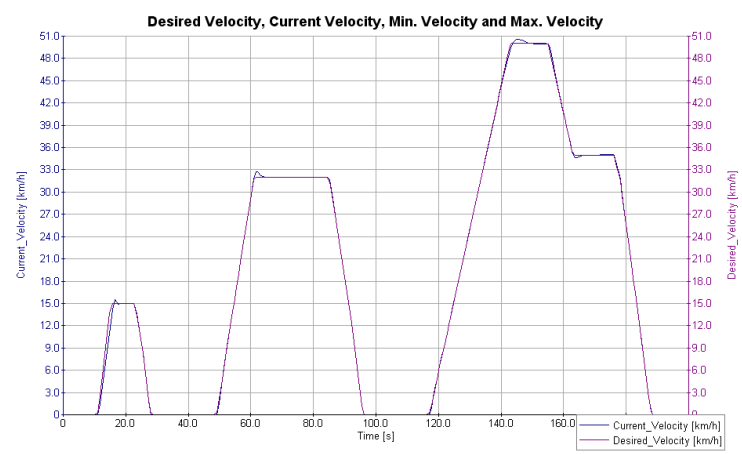

Fig.5 Actual velocity and desired velocity in UDC cycle 


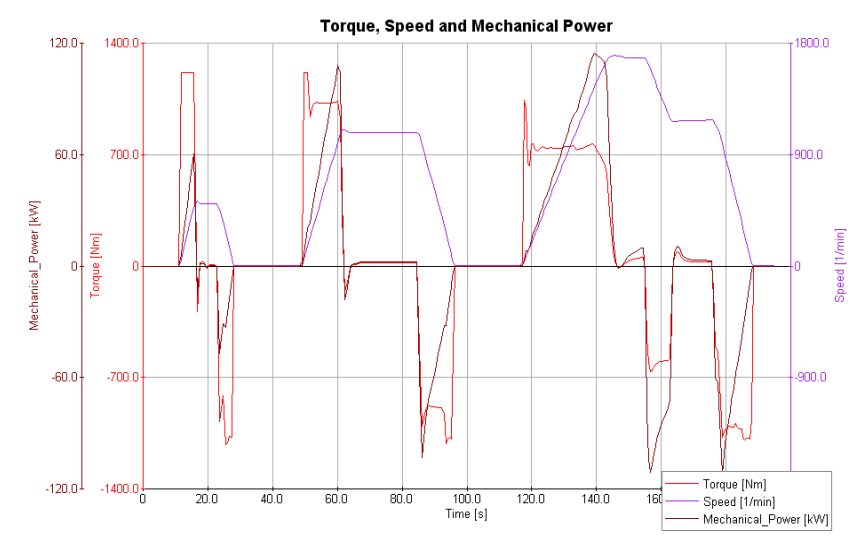

Fig.6 Torque, speed and mechanical power of improved parallel braking

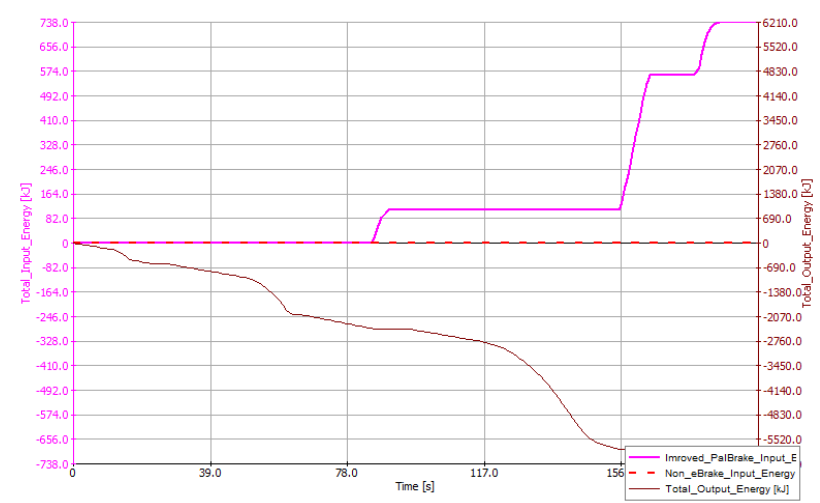

Fig.7 Energy recovery and consumption

As shown in Fig.7, the consumed energy is $6162 \mathrm{KJ}$ in both the improved parallel braking control strategy and non-energy recovery strategy. About $736 \mathrm{KJ}$ energy is recycled by the improved parallel braking control strategy, and the energy recovery rate can reach as much as $11.94 \%$. Therefore, the results prove that the improved parallel braking control strategy can recycle the braking energy effectively.

\section{Conclusions}

For the braking control strategy of range-extended electric commercial vehicle, an improved parallel braking control strategy is proposed in this paper to ensure the braking performance and recycle more energy. Then the simulation model of the improved braking control strategy is established with Cruise and Matlab. Next, the performance of the control strategy is verified by the UDC cycle. Simulation results show that the improved parallel braking control strategy proposed in this paper can optimize the braking force distribution, maximize the braking energy recovery rate on the premise of the best braking performance. And vehicle economy is also improved as well as the braking performance. But the braking force characteristic for improved parallel braking is different from friction braking, so how to control the motor braking force effectively through the electronic control unit will be the direction of further research.

\section{Acknowledgements:}

This work has been supported by NSFC(51507013), Natural science basic research project in Shaanxi Province(2014JQ7269) and Fundamental Research Funds for the Central Universities (310822151025, 310822161002).

\section{References}

[1] Hannan M A, Azidin F A, Mohamed A: Hybrid electric vehicles and their challenges: A review. Renewable and Sustainable Energy Reviews, Vol. 29 (2014), p. 135-150. 
[2] Zhao Guozhu. Research on Braking Stability of Regenerative Braking System in EV and HEV. Nanjing: Nanjing University of Aeronautics and Astronautics(2006).

[3] Tate E D, Harpster M O, Savagian P J. The electrification of the automobile: from conventional hybrid, to plug-in hybrids, to extended-range electric vehicles[J]. SAE international journal of passenger cars-electronic and electrical systems, Vol. (2008), p . 156-166.

[4] Yu Zhisheng. Vehicle Theory[M]. Beijing: Tsinghua University Press(2009).

[5] Zhang Xin,LIU Li,YU Haisheng. Simulation for Regenerative Characteristics of Hybrid Electric Vehicle Braking System. China Journal of Highway and Transport, Vol. 19 (2006),p. 111-116.

[6] Nian X, Peng F, Zhang H. Regenerative Braking System of Electric Vehicle Driven by Brushless DC Motor. IEEE Transactions on Industrial Electronics, Vol. 61(2014), p.5798-5808.

[7] Dong Bing. Energy Management System control Strategy and optimization for Pure Electric Vehicle Based on the Lithium-Ion Power Battery. Changchun: Jilin University(2014). 